\title{
Constant Crunch Coordinates for Black Hole Simulations
}

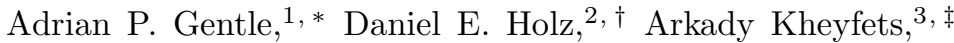

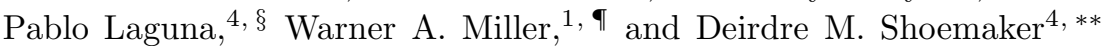 \\ ${ }^{1}$ Theoretical Division (T-6, MS B288), Los Alamos National Laboratory, Los Alamos, NM 87544 \\ ${ }^{2}$ Institute for Theoretical Physics, University of California, Santa Barbara, CA 93106 \\ ${ }^{3}$ Department of Mathematics, North Carolina State University, Raleigh, NC 27695-8205 \\ ${ }^{4}$ Department of Astronomy and Astrophysics and Center for Gravitational \\ Physics and Geometry, Penn State University, State College, PA 16802
}

(Dated: October 30, 2018)

\begin{abstract}
We reinvestigate the utility of time-independent constant mean curvature foliations for the numerical simulation of a single spherically-symmetric black hole. Each spacelike hypersurface of such a foliation is endowed with the same constant value of the trace of the extrinsic curvature tensor, $K$. Of the three families of $K$-constant surfaces possible (classified according to their asymptotic behaviors), we single out a sub-family of singularity-avoiding surfaces that may be particularly useful, and provide an analytic expression for the closest approach such surfaces make to the singularity. We then utilize a non-zero shift to yield families of $K$-constant surfaces which (1) avoid the black hole singularity, and thus the need to excise the singularity, (2) are asymptotically null, aiding in gravity wave extraction, (3) cover the physically relevant part of the spacetime, (4) are well behaved (regular) across the horizon, and (5) are static under evolution, and therefore have no "grid stretching/sucking" pathologies. Preliminary numerical runs demonstrate that we can stably evolve a single spherically-symmetric static black hole using this foliation. We wish to emphasize that this coordinatization produces $K$-constant surfaces for a single black hole spacetime that are regular, static and stable throughout their evolution.
\end{abstract}

\section{CONSTANT CRUNCH SURFACES}

In this paper, we address a single question: Is there a numerically-viable coordinatization of a Schwarzschild black hole spacetime foliated by hypersurfaces of constant (not necessarily zero) mean extrinsic curvature? In other words, can we coordinatize the Schwarzschild spacetime with constant mean extrinsic curvature $(\operatorname{Tr}(K)=$ constant $)$ hypersurfaces so as to bound the growth of metric components and their gradients? We demonstrate here that the single shift freedom yields a spacetime metric that is static, and therefore bounds the growth in time of such gradients. A more complete analysis of the stability of our coordinatization, and a more thorough canvassing of the parameter space, will appear elsewhere. [1] Our foliation is consistent with that of Iriondo et al. [2], who provided a generic constant mean curvature (CMC) foliation of the Reissner-Nordström spacetime for the purpose of finding trapped surfaces. In this paper we focus on the utility of CMC slicings for the numerical simulation of black holes, in support of the emerging field of gravity-wave astrophysics.

The trace of the extrinsic curvature tensor $\left(\operatorname{Tr}(\mathbf{K})=K^{a}{ }_{a}=K\right)$ at a point on a spacelike hypersurface measures the fractional rate of contraction of 3-volume along a unit normal to the surface. It represents the amount of "crunch" the 3-surface is experiencing at the point, at a given time. If all the observers throughout a spacelike hypersurface moving in time orthogonal to the surface experience the same amount of contraction per unit proper time, we say that the surface is a $K$-surface or a "constant crunch" surface. In this paper we examine foliations of a single sphericallysymmetric, static black hole where each spacelike hypersurface has the same constant value of the extrinsic curvature, $K$.

Generic $K$-surface foliations have found great utility in the numerical simulation of cosmological spacetimes. [3] In addition to decoupling the three momentum constraint equations from the Hamiltonian constraint, these surfaces (in the case of compact or $\mathrm{W}$-model universes) provide a convenient cosmological time parameter ( $K$, or York, time). [4] Furthermore, for such cosmological spacetimes one has powerful existence and uniqueness theorems. [5, 6] Extensive

\footnotetext{
*Electronic address: apg@lanl.gov

$\dagger$ Electronic address: deholz@itp.ucsb.edu

‡Electronic address: khevfets@math.ncsu.edu

$\S$ Electronic address: pablo@astro.psu.edu

IElectronic address: wamalan gov

${ }^{* *}$ Electronic address: deirdre@astro.psu.edu
} 
work into the characteristics of these surfaces for Schwarzschild spacetimes has been done by Brill et al., [7] and foundational work into their use in numerical relativity was done by D. Eardley et al.. [8] More recently Pervez et al. [9] provided a foliation partially covering the Schwarzschild spacetime with $K$-surfaces, with $K$ ranging from $-\infty$ to $\infty$, and Iriondo et al. [2] provided a generic constant mean curvature foliation of the Reissner-Nordström spacetime for the purpose of finding trapped surfaces. In this paper we build upon the work of these investigators by examining the utility of these surfaces for numerical relativity in support of gravity-wave detectors.

Although surfaces of constant $K$ were thoroughly investigated decades ago, their use in current numerical simulations of black holes is conspicuously absent (apart from the use of maximal $(K=0)$ surfaces). [10] One reason why these slicing methods have not been more fully developed is that they lag in time close in, to avoid crashing into the singularity, while they simultaneously evolve forward normally at the outer edge of the grid to allow for wave extraction. This tension, many fear, will unavoidingly lead to unbounded growth in the metric and extrinsic curvature components in the intermediate region, as is indeed found in maximal slicing. This computational concern has been referred to by the numerical relativity community as "grid stretching" or "grid sucking." We show in this paper that a proper choice of radial shift can yield a constant crunch foliation of a spherically-symmetric black hole without such pathologies. In fact, we foliate a Schwarzschild black hole such that the 3-metric and extrinsic curvature are both bounded and static (i.e. unchanging in time).

To numerically evolve a black hole 3 -space in time it is desirable to have a foliation, and its coordinatization, which satisfy the following four properties:

1. Avoids black hole singularities or facilitates their excision.

2. Possesses asymptotically null hypersurfaces to aid in radiation extraction.

3. Minimizes steep gradients in the lapse, shift, 3-metric and extrinsic curvature tensor.

4. Maximizes the future development of the initial data for the purpose of gravity-wave extraction.

As a first step towards achieving these goals for systems containing multiple black holes, we explore the families of $K$-surfaces in the Schwarzschild spacetime, and find a CMC foliation satisfying the above properties.

In the next section we construct the $K$-surfaces outside and inside the horizon. In section III we explore the properties of the $K$-surfaces, dwelling in particular on their approaches to the singularity. We also examine and illustrate the three families of $K$-surfaces. In section IV] we derive a metric for Schwarzschild whose constant time slices are $K$-surfaces. We restrict our attention to a subfamily of $K$-surfaces - surfaces which, when generalized to the colliding black hole spacetimes, support the gravity wave detection problem. We also present some preliminary numerical simulations using constant-crunch coordinates. We conclude with general comments on the applicability of $K$-surfaces to numerical calculations of more general black hole spacetimes.

\section{CONSTRUCTION OF CONSTANT CRUNCH SURFACES}

Over three decades ago Eardley and Smarr [8] carried out a generic classification of the spacetimes that could be simulated numerically, and investigated the limitations that the presence of singularities would impose. In their paper they argued that CMC slicings are particularly useful for numerical purposes. In particular, they demonstrated this explicitly by constructing numerical solutions to a wide array of dust collapse models. In a similar vein, Brill et al. [7] explored the nature of CMC slices of the Schwarzschild spacetime, and they also presented some numerical examples. In the present work, we explore the numerical utility of CMC slicings in the case of single black hole spacetimes. For the sake of clarity, we will commence with a re-derivation of the equations governing the CMC surfaces starting from Schwarzschild coordinates. From there, we will explore specific properties of the surfaces, paying particular attention to implications for numerical relativity.

We wish to find a spacelike hypersurface in the Schwarzschild spacetime such that every point on the surface has the same constant value of the trace of the extrinsic curvature tensor. We have at our disposal the specification of the initial-value data, as well as the freedom to choose the lapse and shift throughout the evolution. To begin, let us take the standard coordinate system of a single black hole spacetime of mass $M$ in Schwarzschild coordinates:

$$
d s^{2}=-B(r) d t^{2}+C(r) d r^{2}+r^{2}\left(d \theta^{2}+\sin ^{2} \theta d \varphi^{2}\right)
$$

with $B(r)=(1-2 M / r)$ and $C(r)=1 / B(r)$. It will be convenient to treat separately the regions inside and outside of the horizon. We will find that the two are related by an isometry. 


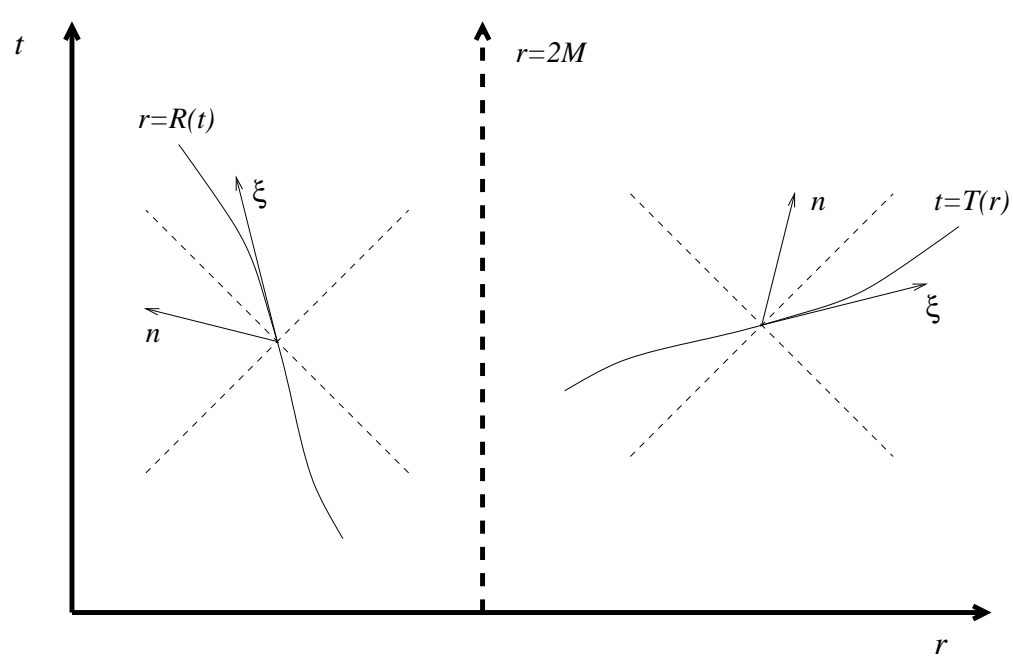

FIG. 1: To construct CMC slices, at each point we introduce a unit normal vector $\mathbf{n}$ and the unit tangent vector $\boldsymbol{\xi}$ to the surface. The local light cones are depicted by the light dashed lines.

\section{A. Outside the Horizon $(r>2 M)$}

Outside the horizon, CMC surfaces will be labeled by $t=T(r)$ (Fig. 1). The requirement that the trace of the extrinsic curvature be constant throughout this surface yields a first order differential equation for $T(r)$, determined by examining the behavior of the normals to the surface. The normal $\mathbf{n}$ to the spacelike hypersurface $T$ is given by,

$$
\mathbf{n}=N_{0} \nabla(t-T(r))=n_{t} d t+n_{r} d r=N_{0}\left(d t-T^{\prime} d r\right),
$$

where $N_{0}$ is a normalization constant and primes denote differentiation with respect to $r$. The normalization is fixed by demanding that

$$
\begin{aligned}
\mathbf{n} \cdot \mathbf{n} & =-1 \\
& =g^{t t} n_{t} n_{t}+g^{r r} n_{r} n_{r} \\
& =N_{0}^{2}\left(-\frac{1}{B}+\frac{1}{C} T^{2}\right) .
\end{aligned}
$$

Therefore,

$$
N_{0}=-\frac{1}{\sqrt{C-B T^{\prime 2}}}
$$

and

$$
\begin{aligned}
& n_{r}=\frac{T^{\prime}}{\sqrt{C-B T^{\prime 2}}}, \\
& n_{t}=-\frac{1}{\sqrt{C-B T^{\prime 2}}} .
\end{aligned}
$$

The contravariant components of the normal are given by

$$
\begin{aligned}
& n^{r}=g^{r r} n_{r}=\frac{T^{\prime}}{C \sqrt{C-B T^{\prime 2}}}, \\
& n^{t}=g^{t t} n_{t}=\frac{1}{B \sqrt{C-B T^{\prime 2}}} .
\end{aligned}
$$

The trace of the extrinsic curvature is the fractional rate of contraction of 3-volume per unit proper time along the normal, namely

$$
K=-n_{; \alpha}^{\alpha}=-\frac{1}{r^{2}} \frac{d}{d r}\left(r^{2} n^{r}\right)
$$


Substitution of $n^{r}$ into Eq. (11) yields a second-order ordinary differential equation for $T$. Integrating this equation, we find

$$
H=\left(\frac{B r^{2} T^{\prime}}{\sqrt{C-B T^{\prime 2}}}\right)+J
$$

with $H$ an integration constant and $J$ an indefinite integral given by

$$
J=\int^{r} K \sqrt{B C} r^{2} d r=\frac{1}{3} K r^{3} .
$$

Along the surface the rate of change of proper time, $d \tau$, with proper distance, $d s$, is related to the slope of the surface $T$,

$$
\frac{d \tau}{d s}=\sqrt{\frac{B}{C}} T^{\prime}
$$

From Eq. (12) we find

$$
\left(\frac{d \tau}{d s}\right)^{2}=\frac{(H-J)^{2}}{(H-J)^{2}+B r^{4}}
$$

\section{B. Inside the Horizon $(r<2 M)$}

Finding the $K$-constant slices of Eq. (1) within the horizon is similar to the calculation done in the previous section; however, as the roles of time and space coordinates reverse within the horizon, we will find it useful to parameterize our spacelike surface as a function of coordinate $t$, and look for $K$-constant surfaces of the form (Fig. 1)

$$
r=R(t) .
$$

The normal $\mathbf{n}$ to the spacelike hypersurface $R$ is given by

$$
\mathbf{n}=N_{0} \nabla(R(t)-r)=n_{t} d t+n_{r} d r=N_{0}(\dot{R} d t-d r),
$$

with differentiation with respect to $t$ denoted by dots and the $N_{0}$ a normalization constant fixed by:

$$
\begin{aligned}
\mathbf{n} \cdot \mathbf{n} & =-1 \\
& =g^{t t} n_{t} n_{t}+g^{r r} n_{r} n_{r} \\
& =N_{0}^{2}\left(\frac{1}{C}-\frac{1}{B} \dot{R}^{2}\right) .
\end{aligned}
$$

We have, therefore,

$$
N_{0}=\frac{-1}{\sqrt{C \dot{R}^{2}-B}}
$$

and

$$
\begin{aligned}
& n_{r}=\frac{1}{\sqrt{C \dot{R}^{2}-B}}, \\
& n_{t}=\frac{-\dot{R}}{\sqrt{C \dot{R}^{2}-B}} .
\end{aligned}
$$

The contravariant components of the normal are given by

$$
\begin{aligned}
& n^{r}=g^{r r} n_{r}=\frac{1}{C \sqrt{C \dot{R}^{2}-B}}, \\
& n^{t}=g^{t t} n_{t}=\frac{\dot{R}}{B \sqrt{C \dot{R}^{2}-B}} .
\end{aligned}
$$


From Eq. (11), we once again find that fixing the trace of the extrinsic curvature gives us a second-order differential equation for $\vec{R}(t)$, namely,

$$
K=\frac{-2}{C R \sqrt{C \dot{R}^{2}-B}}-\frac{2 C B^{\prime} \dot{R}^{2}-B\left(B^{\prime}+C^{\prime} \dot{R}^{2}+2 C \ddot{R}\right)}{2\left(C \dot{R}^{2}-B\right)^{3 / 2}}
$$

which can be simplified to

$$
K R^{2} \dot{R}=-\frac{d}{d t}\left(\frac{B R^{2}}{\sqrt{C \dot{R}^{2}-B}}\right) .
$$

Paralleling the approach from the last section, we introduce an integration constant, $H$, and an indefinite integral $J$ (given by Eq. (13)), to obtain the first integral:

$$
H=\left(\frac{B R^{2}}{\sqrt{C \dot{R}^{2}-B}}\right)+J .
$$

From Eq. (27) we find that the "proper velocity" along the surface, $d s / d \tau=\sqrt{C / B} \dot{R}$, results in the same equation both inside and outside of the horizon (Eq. (15)). This can be rewritten as

$$
\left(\frac{d s}{d \tau}\right)^{2}=1+\frac{B R^{4}}{(H-J)^{2}}
$$

The spacelike $K$-surfaces obtained from the first integrals, Eqs. (15) and (29), differ only by an isometry,

$$
T^{\prime} \Longleftrightarrow \frac{1}{\dot{R}}
$$

\section{PROPERTIES OF THE $K$-SURFACES}

The spatial metric of a $K$-surface outside of the horizon is given by

$$
\begin{aligned}
d s^{2} & =d \ell^{2}+r^{2} d \Omega^{2} \\
& =\left(C-B T^{\prime 2}\right) d r^{2}+r^{2} d \Omega^{2} .
\end{aligned}
$$

Within the horizon it becomes

$$
\begin{aligned}
d s^{2} & =d \ell^{2}+r^{2} d \Omega^{2} \\
& =\left(C \dot{R}^{2}-B\right) d t^{2}+r^{2} d \Omega^{2} .
\end{aligned}
$$

These two expressions differ by the isometry of Eq. (30). Using Eq. 15. we can rewrite them in terms of $H$ and $K$ :

$$
d s^{2}=\frac{r^{4}}{(H-J)^{2}+B r^{4}} d r^{2}+r^{2} d \Omega^{2} .
$$

From this we arrive at the scalar curvature of the $K$-surface:

$$
{ }^{(3)} R=-\frac{2}{3} K^{2}+\frac{6 H^{2}}{r^{6}} \text {. }
$$

Similarly, by using Eqs. (15) and (29), the extrinsic curvature associated with observers moving on world lines orthogonal to the $K$-slices are also expressible in terms of $K$ and $H$ :

$$
\begin{aligned}
& K_{\hat{r}}^{\hat{r}}=\frac{1}{3} K+\frac{2 H}{r^{3}}, \\
& K_{\hat{\theta}}^{\hat{\theta}}=K_{\hat{\phi}}^{\hat{\phi}}=\frac{1}{3} K-\frac{H}{r^{3}} .
\end{aligned}
$$


Schwarzschild
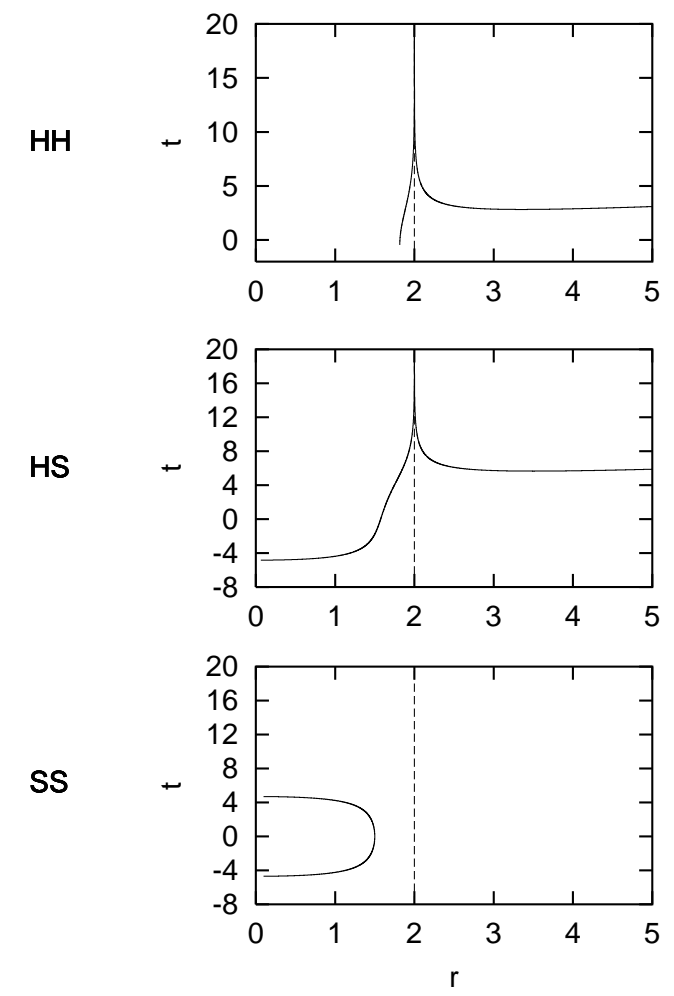

Kruskal-Szekeres
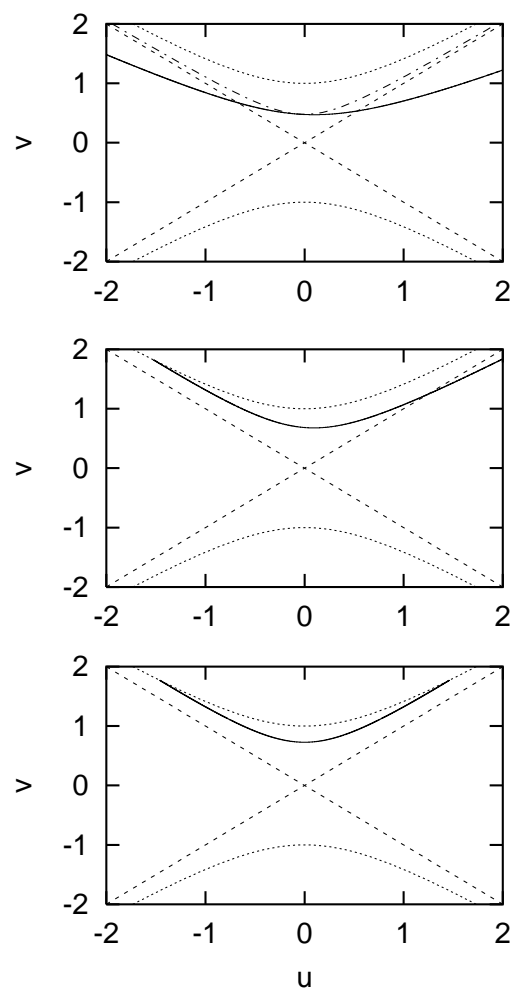

FIG. 2: An example of the three families of spacelike $K$-surfaces for $K=-0.1$. The first row $(H H)$ depicts a representative horizon-to-horizon surface using $H=-1.25$, which corresponds to $R_{\min } \approx 1.816$. This spacelike hypersurface is represented both in Schwarzschild coordinates (left column) and in Kruskal-Szekeres coordinates (right column). The middle two graphs are the horizon-to-singularity $(H S)$ surfaces using $H=-1.43$. The bottom two graphs represents a typical singularity-tosingularity $(S S)$ surface. We have used $H=-1.25$ to generate this $S S K$-surface. The $H H$ and $S S$ surfaces are close to their critical radii $\left(R_{c} \approx 1.5646\right)$ and therefore appear flattened, as described in the text.

The $K$-surfaces are therefore parametrized by two constants: the trace of the extrinsic curvature tensor, $K$, and the constant of integration, $H$. In addition one must fix a single point on the surface, $t_{o}=T\left(r_{o}\right)$, which amounts to setting a time translation parameter. As can be seen in Eqs. (36)-(38), the constant $H$ controls the variation of the intrinsic and extrinsic curvatures over the $K$-surface.

To elucidate the nature of the $K$-surfaces, we numerically integrate Eqs. (15) and (29). We find that within the horizon there are 3 classes of $K$-constant surfaces, differentiated by their asymptotic behavior. The singularitysingularity surfaces $(S S)$ begin at the singularity aligned with the null surface, reach up towards the horizon, and then fall back, reaching the singularity along the null cone. The horizon-horizon surfaces $(H H)$, which we have also dubbed "horizon-hugging" surfaces, asymptote to the horizon ( $r \rightarrow 2 M$ for $|t| \rightarrow \infty$ in Schwarzschild), dipping down towards the singularity in between. This feature was previously remarked upon by Brill et al. [7]. The asymptotes converge toward a null surface at the horizon. Finally, the horizon-singularity $(H S)$ surfaces begin at the horizon, and asymptote in to the singularity. Representative surfaces for the value $K=-0.1$ are shown in Fig. 2. We integrate the $H H$ and $H S$ surfaces across the horizon into the region $r>2 M$ by imposing continuity of the surface and its first derivative at the horizon. Because of the isometry, Eq. (30), the surfaces outside of the horizon are characteristically similar to those on the inside; in particular, both sets are null at their asymptotes.

We have chosen to use the acronym $H H$ for the horizon-to-horizon hypersurfaces, in lieu of referring to them as "regular" hypersurfaces [7], as each of the three types of $K$-surfaces are, in a strict sense, regular. In particular, each surface asymptotes to a null surface, be it at the singularity or the horizon. Observers on such a surface, or more precisely, observers that are time synchronized throughout the surface, are never seen crossing the horizon, nor do they ever reach the singularity! Outside the horizon, every $H H$ and $H S \mathrm{~K}$-surface $(K \neq 0)$ asymptotes for large $r$ to null infinity. $K$-surfaces corresponding to positive values of $K$ asymptote to past null infinity, and asymptote to future null infinity for $K<0$.

To gain a qualitative understanding of the $K$-constant foliation, it is useful to analyze Eq. (29) as an energy 


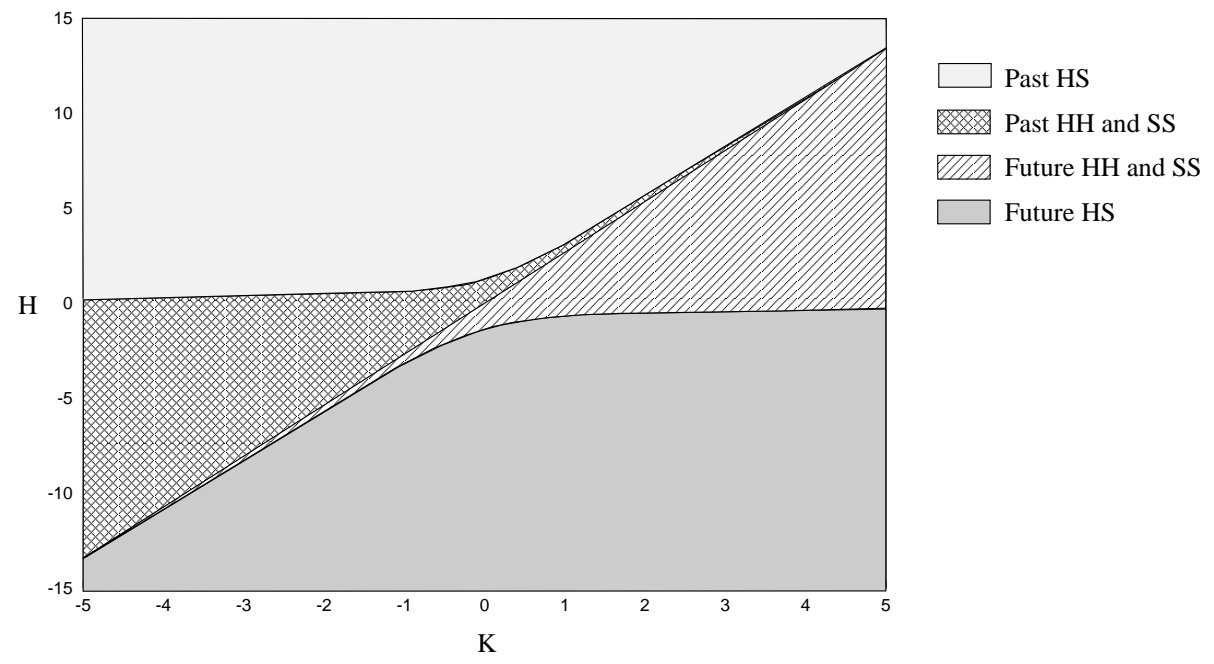

FIG. 3: Phase space diagram of the classification of $K$-surfaces. The $K$-surfaces have been classified into 3 groups according to their asymptotic behavior (see text). Which class a particular surface falls into depends upon the values of $K$ and $H$, as detailed in the figure. For the purposes of numerics, the surfaces of interest are to be found in the "Future $H H$ and $S S$ " wedge, with $K<0$ (see section [II]).

conservation equation for a particle of unit total energy $(E=1)$ moving in the potential

$$
V(r)=\frac{-\left(1-\frac{2 M}{r}\right) r^{4}}{\left(H-\frac{1}{3} K r^{3}\right)^{2}}
$$

By using this energy equation we can determine the closest approach to the singularity, $R_{\text {min }}$, of a given $H H K$ constant surface. Two conditions must be satisfied to determine $R_{\min }$. First, the closest approach occurs when $\dot{R}=(d s / d \tau)=0$, which is equivalent to demanding

$$
V\left(R_{\min }\right)=1 .
$$

This condition leads to a sixth-order polynomial in $R_{\min }$ :

$$
\frac{K^{2}}{9} R_{\min }^{6}+R_{m i n}^{4}-2\left(M+\frac{H K}{3}\right) R_{m i n}^{3}+H^{2}=0 .
$$

Second, the solution for this surface at $R\left(t_{\min }\right)=R_{\min }$ must be concave, so as to rule out the $S S$ surfaces (which bend towards the singularity rather than the horizon). This is enforced by demanding:

$$
\left.\ddot{R}\left(t_{\min }\right)\right|_{\dot{R}\left(t_{\min }\right)=0}=\frac{\left(R_{\min }-2\right)\left[-3+2 R_{\min }+K R_{\min }^{2} \sqrt{\frac{2}{R_{\min }}-1}\right]}{R_{\min }^{3}} \geq 0 .
$$

The solution of these two conditions, as shown in Fig. 3, gives rise to the emergence of two critical values for $H$, namely $H_{ \pm}$, for a given value of $K$. In addition, an $H H$ surface can be made to approach arbitrarily closely to the singularity at $r=0$ by choosing an appropriately large positive value of $K$. Negative values of $K$ tend to "hug the horizon."

For fixed $K$ and $H$ we know how to compute how closely a CMC surface comes to the singularity. But, for a given value of $K$, what value of $H$ gives the closest overall approach to the singularity? We can determine the critical values for $R$ and $H$, given by $R_{c}$ and $H_{ \pm}$respectively, by looking at the point where the first and second time derivatives of $R(t)$ vanish. $H_{-}$occurs along the lower boundary of the contour plot in Fig. 急, and corresponds to the $K$-surface that reaches down the furthest towards the singularity for a given value of $K$. The vanishing of $\left.\ddot{R}\right|_{\dot{R}=0}$ leads to the following equation for $R_{c}$ :

$$
\left(R_{c}-2\right)\left(-3+2 R_{c}+K R_{c}^{2} \sqrt{\frac{2}{R_{c}}-1}\right)=0
$$




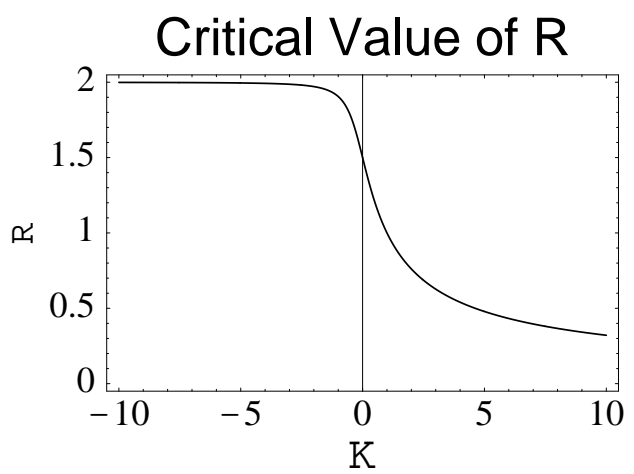

FIG. 4: Critical value of $R_{c}=R_{-}$as a function of $K$ for Schwarzschild coordinates and $M=1$. One can readily see that the $K$-surface "hugs" the horizon for large negative values of $K$.

which can be rewritten as a 4 th order polynomial in $R_{c}$

$$
K^{2} R_{c}^{4}-2 K^{2} R_{c}^{3}+4 R_{c}^{2}-12 R_{c}+9=0 .
$$

One must take care in examining the roots of this equation, as there are more solutions to Eq. (44) than there are for Eq. (43). Nevertheless this equation gives two distinct real roots, depending on the sign of $K$ :

$$
\begin{aligned}
& \left.R_{c}\right|_{K>0}=\frac{1}{2}-\frac{1}{2} \sqrt{3-\frac{8}{K^{2}}-\chi+\frac{2}{\sqrt{\chi}}+\frac{16}{K^{2} \sqrt{\chi}}}+\frac{\sqrt{\chi}}{2}, \\
& \left.R_{c}\right|_{K<0}=\frac{1}{2}+\frac{1}{2} \sqrt{3-\frac{8}{K^{2}}-\chi+\frac{2}{\sqrt{\chi}}+\frac{16}{K^{2} \sqrt{\chi}}}+\frac{\sqrt{\chi}}{2}
\end{aligned}
$$

where

$$
\begin{aligned}
\xi & \equiv 32+108 K^{2}+243 K^{4}+27 K^{2} \sqrt{16+56 K^{2}+81 K^{4}}, \\
\chi & \equiv 1-\frac{8}{3 K^{2}}+\frac{16+36 K^{2}}{32^{1 / 3} K^{2} \xi^{1 / 3}}+\frac{2^{1 / 3} \xi^{1 / 3}}{3 K^{2}} .
\end{aligned}
$$

When $K=0$ we see from Eq. (43) that $R_{c}=3 / 2$. The regular $K$-surfaces are thus bounded between the horizon at $R_{+}=2 M$ and $R_{-}=R_{c} M$. Using Eq. (28), and setting $\dot{R}=0$, we obtain, for the case of a black hole in Schwarzschild coordinates

$$
H_{ \pm}=\frac{B_{ \pm} R_{ \pm}^{2}}{\sqrt{-B_{ \pm}}}+\frac{K R_{ \pm}^{3}}{3}
$$

with

$$
B_{ \pm}=\left(1-\frac{2 M}{R_{ \pm}}\right)
$$

We therefore have

$$
H_{+}=\frac{8}{3} M^{3} K
$$

For large values of $|K|$ one can show that the critical value of $R, R_{-}$, depends upon the sign of $K$. In particular,

$$
R_{c} \longrightarrow \begin{cases}2-\frac{1}{8} K^{-2} & \text { for } K \ll-1 \\ \left(\frac{9}{4}\right)^{1 / 3} K^{-2 / 3} & \text { for } K \gg 1\end{cases}
$$

This in turn gives the following asymptotic values for $H_{m}$ :

$$
H_{-} \longrightarrow \begin{cases}-\frac{8}{3} K-\frac{1}{2 K} & \text { for } K \ll-1 \\ \frac{-9}{2 K} & \text { for } K \gg 1\end{cases}
$$




\section{CONSTANT CRUNCH COORDINATES: A SPACETIME METRIC FOR A $K$-SURFACE FOLIATION OF THE SCHWARZSCHILD BLACK HOLE.}

A number of features of the $K$-constant surfaces presented in the previous sections seem particularly well suited to the numerical analysis of generic black hole spacetimes. First, the surfaces asymptote to a null surface, making them effective for gravity wave extraction. Second, the $K$-surfaces naturally avoid the crushing singularity. Finally, for large negative values of $K$ the surfaces "hug the horizon." This last feature, illustrated in Fig. 6 , allows one to focus attention on the region relevant for gravity wave generation - the region outside the horizon.

In this section we generate a $K$-constant foliation for the Schwarzschild black hole that, in addition to the properties just mentioned, also has regular and static metric and extrinsic curvature components. To generate this $K$-constant slicing we use the coordinate transformation

$$
\begin{aligned}
\bar{t} & =t-T(r), \\
\rho & =r .
\end{aligned}
$$

Under this transformation, the metric from Eq. (11) becomes

$$
d s^{2}=-\left(1-\frac{2 M}{\rho}\right) d \bar{t}^{2}+2 \frac{(J-H)}{\sqrt{(H-J)^{2}+\left(1-\frac{2 M}{\rho}\right) \rho^{4}}} d \bar{t} d \rho+\frac{\rho^{4}}{(H-J)^{2}+\left(1-\frac{2 M}{\rho}\right) \rho^{4}} d \rho^{2}+\rho^{2} d \Omega^{2} .
$$

The constant $\bar{t}$ slices of this metric are $K$-constant surfaces. It is to be noted that Eq. (56) agrees with Eq. (53) of Iriondo et al. (for the case of constant $K$ and vacuum). [2] However, in order to regularize the $g_{\rho \rho}$ metric component at the throat, we add the isotropic-like radial transformation [11:

$$
\bar{r}=\frac{1}{2}\left(-\frac{1}{2} R_{\min }+\rho+\sqrt{-R_{\min } \rho+\rho^{2}}\right),
$$

with $R_{\min }$ the minimum coordinate location of the throat, given by Eq. (41). This coordinate representation of a black hole spacetime provides a foliation with $K$-constant, $H$-constant spacelike hypersurfaces. Each hypersurface is metrically equivalent to all others - the surfaces are independent of $\bar{t}$, and hence static. In addition, the hypersurfaces are asymptotically null $\left(T^{\prime}(r) \rightarrow 1\right.$ as $\left.r \rightarrow \infty\right)$. Furthermore, the lapse, the shift, and all of the 3-metric and extrinsic curvature components are regular and well behaved, as illustrated in Fig. f.

In addition to restricting ourselves to the singularity-avoiding family $(H H)$ of $K$-surfaces, two additional conditions on the $K$-surfaces are demanded by the nature of our problem - the eventual simulation of the gravity-wave emission from two interacting black holes. First, the foliations must asymptote at large $\bar{r}$ to future null infinity. Therefore, we must restrict our attention to negative values for the trace of the extrinsic curvature tensor, $K$. Second, we require that such negative $K$ hypersurfaces enter the future singularity region. This will ensure proper coverage of the relevant region just above the future horizon, which is precisely where the gravity waves are produced. However, we expect the initial-data formulation for such surfaces to be involved, and this may guide our choices even more systemically. The two additional requirements limit us to the relatively narrow wedge of Fig. 3, formed by restricting to the "Future $H H$ and $S S^{\prime \prime}$ shaded region with $K<0$.

A representative constant-crunch foliation generated by Eq. (56) is shown in Fig. 6. The avoidance of "grid stretching" is accomplished by a suitable choice of shift vector. To illustrate the non-zero shift we show the $K=-1$, $H \approx-3.11$ foliation of Schwarzschild in Fig. 8, with the explicit misalignment of the $r=$ constant line segment and the normal vector.

Finally, we describe several preliminary numerical experiments using $K$-surfaces. A full treatment of a single black hole using $K$-constant foliations will be presented elsewhere [1]. Here we present several sample evolutions demonstrating the utility of constant mean curvature slicings. Figures 8 and 9 display results from the simplest possible test of $K$-constant foliation of the Schwarzschild geometry; the domain is taken to be a thin shell close to the horizon (in this case $r \in[1,5]$ ), analytic Dirichlet conditions are applied at both boundaries of the computational domain, and analytic lapse and shift conditions obtained from Eqs. (56) and (57) are used. The figures represent the singularity-avoiding foliation $K=-1$ and $H=-3$, for which the evolution was found to be stable and accurate over very long timescales. Using 50 grid points, the code succesfully ran beyond $t=50,000 \mathrm{M}$ while maintaining high accuracy. The fractional error in the metric components was typically $1-2 \%$.

Fig. 8 shows the convergence of the mean fractional error in the metric component $\bar{a}=\sqrt{g_{\bar{r} \bar{r}}}$ as a function of time. Each curve has been rescaled by a factor of $4^{p}$, where the number of grid points is given by $400 / 2^{p}$, with $p=0,1,2,3$. For $t>2$ the solution is approximately second order accurate. Initially, noise generated on the inner boundary causes fluctuations whose magnitude is largely independent of the number of grid points. Fig. 9 shows snapshots of the 

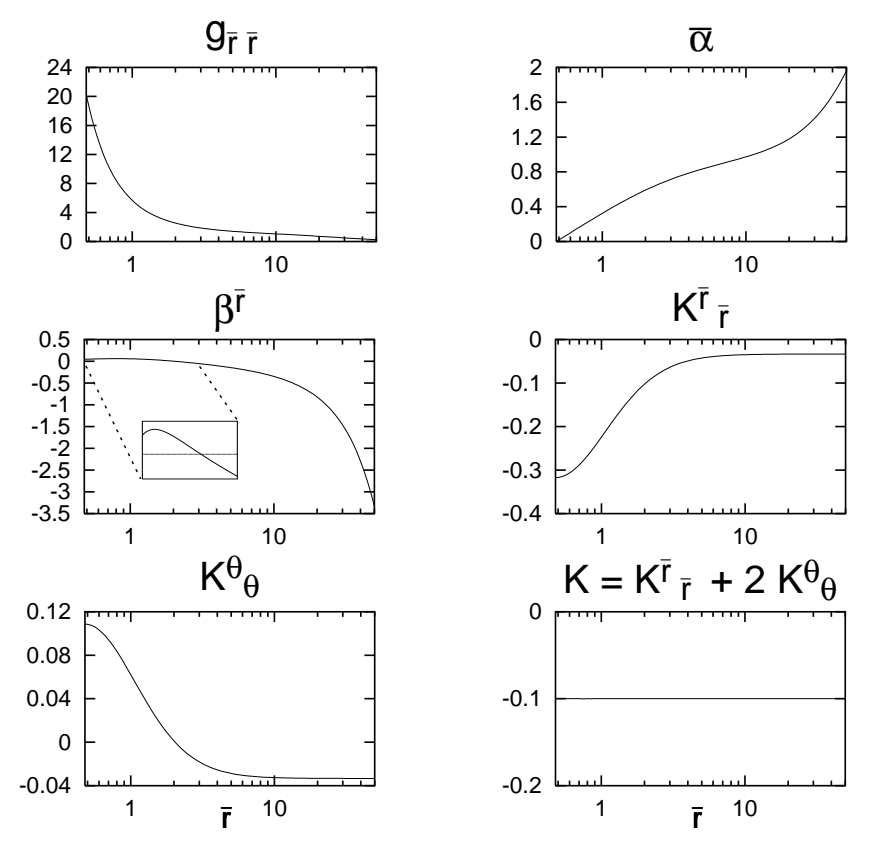

FIG. 5: The radial behavior of the various components of the spacetime metric (Eq. $(\sqrt{50})$ ), under the isotropic-like transformation given in Eq. (57), for $M=1, K=-0.1, H=-1.0$ and $\bar{R}_{\min } \approx 0.47922$. From upper left to lower right we plot the radial metric component $\left(g_{\bar{r} \bar{r}}\right)$, lapse $(\bar{\alpha})$, radial shift $\left(\beta^{\bar{r}}\right)$, diagonal components of the extrinsic curvature tensor $\left(K_{\bar{r}}^{\bar{r}}\right.$ and $\left.K_{\bar{\theta}}^{\bar{\theta}}=K_{\bar{\phi}}^{\bar{\phi}}\right)$, and in the lower right frame the trace of the extrinsic curvature tensor $\left(K=K_{\bar{r}}^{\bar{r}}+K_{\bar{\theta}}^{\bar{\theta}}+K_{\bar{\phi}}^{\bar{\phi}}\right)$ as a consistency check. All of the functions are regular and well behaved. The growth of the lapse and shift for large $\bar{r}$ is expected, as the surfaces become asymptotically null. We show an exploded view of the behavior of the radial shift near the throat to emphasize that the shift changes sign and becomes positive before reaching $\bar{r}=\bar{R}_{m i n}$.
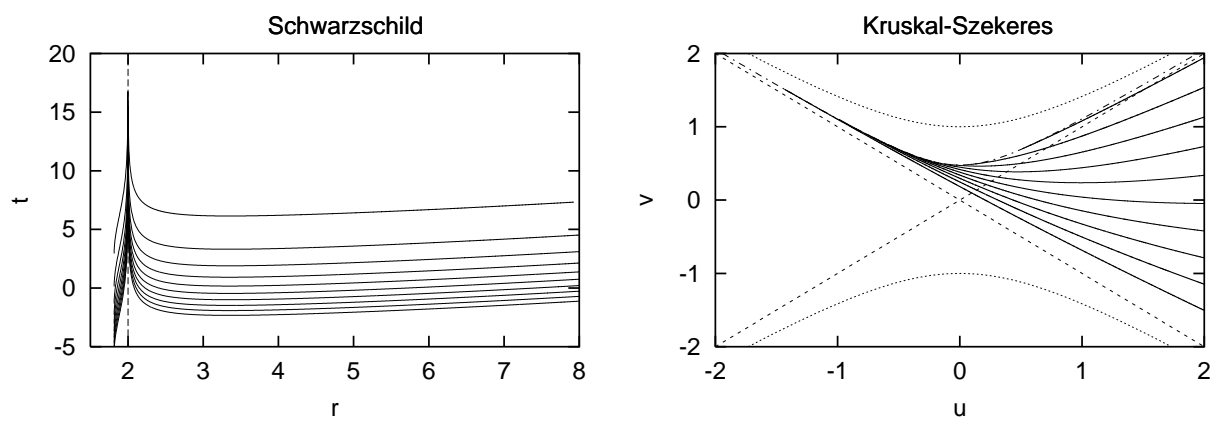

FIG. 6: The constant crunch coordinate foliation of the Schwarzschild spacetime. We show the foliation generated by Eq. (56) for $M=1, K=-0.1, H=-1.25$ and $R_{\min } \approx 1.816$, in Schwarzschild and Kruskal-Szekeres coordinates.

Hamiltonian constraint at various times in the evolution. Qualitatively, a wave which is triggered by truncation error is seen to propagate outwards from the inner boundary. The amplitude reduces rapidly, before growing once more as it is reflected off the outer boundary. The magnitude and speed of propagation of the wave quickly decay as the wave moves back into the domain, leaving a static solution which is stable beyond $t=50,000 \mathrm{M}$. The numerical error which initially propagates through the domain is caused entirely by the analytic Dirichlet boundary conditions, and can be largely eliminated by the use of more realistic conditions. [1]

The numerical runs presented here evolve a $K=-1$ singularity-avoiding hypersurface that asymptotes to null infinity, entering the past singularity region. This is not of the class of $K$-surfaces emphasized in this paper for use in numerical relativity. Ideally, we would have preferred presenting the evolution of a $K=-1 H H$ surface that enters the future singularity region. However, we were unable to find an $H H$ surface in the future singularity region that 

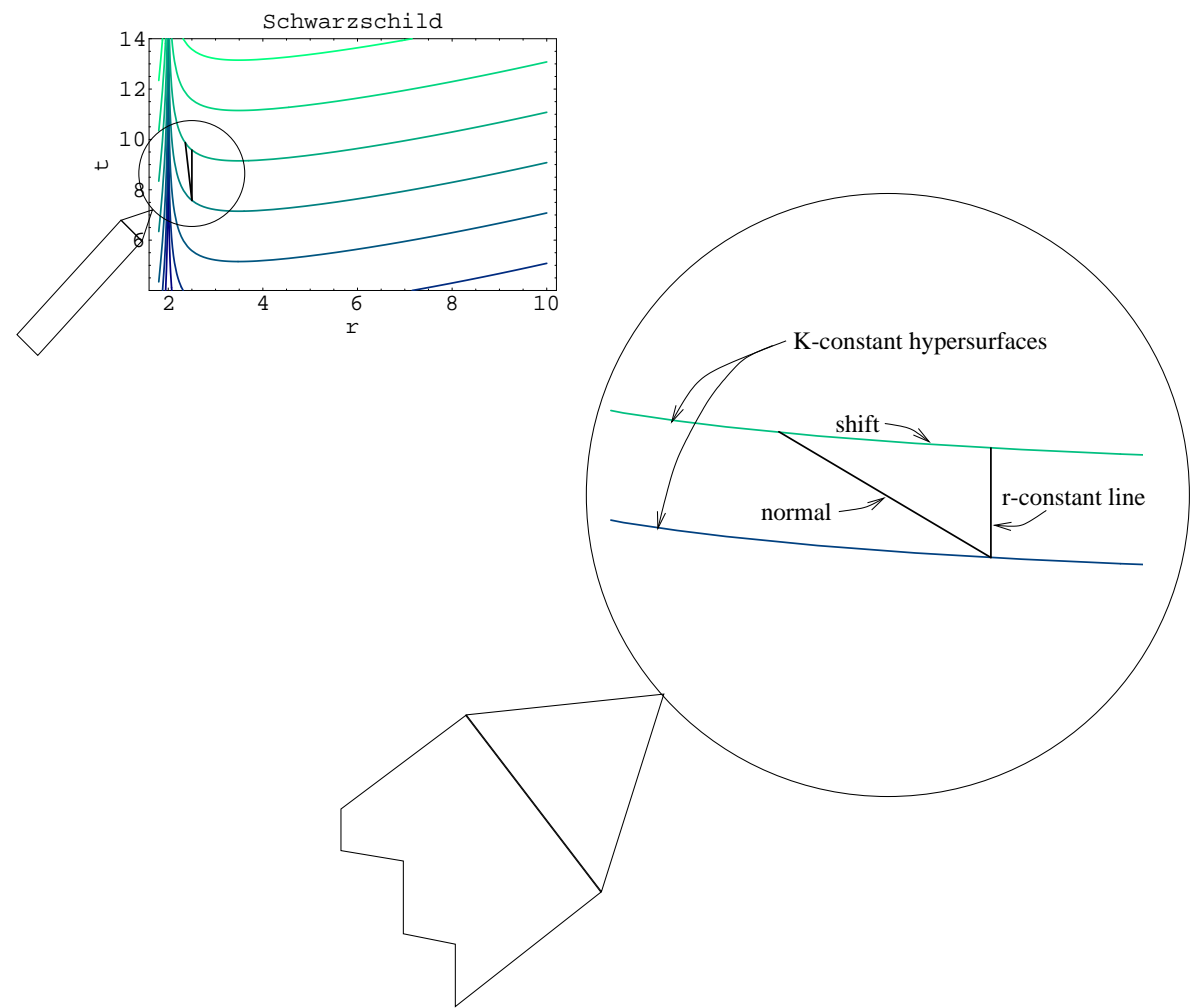

FIG. 7: The non-zero shift in the constant-crunch coordinatization of the Schwarzschild spacetime. We show a foliation generated by Eq. (56) for $M=1, K=-1, H \approx-3.11$, and $R_{\min } \approx 1.9$. We show the $r=$ constant line segment at a point on one of the surfaces, together with the inward-pointing normal vector. The misalignment of these two line segments indicates the non-zero shift in this static coordinatization. A magnified view of the two line segments is shown in the bottom panel.

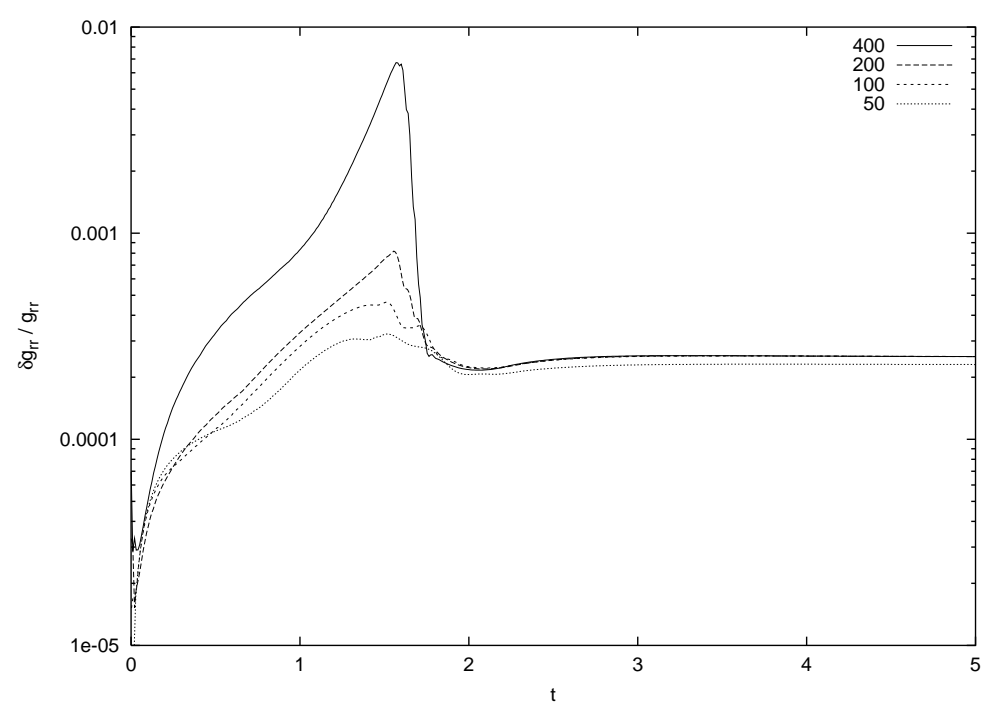

FIG. 8: A numerical example: convergence of the radial metric component for the evolution of an HH surface spanning the past singularity region and reaching positive null infinity. We show the time evolution of a single black hole with $M=1, K=$ $-1, H=-3$, and plot the mean fractional error in the radial metric component $\bar{a}=\sqrt{g_{\bar{r}}}$. Results are shown for four different resolutions, $N=400 / 2^{p}(p=0,1,2,3)$, where each curve has been rescaled by a factor of $4^{p}$. For $t>2$ the solution displays approximately second order convergence, with a fractional error of around $0.1 \%$ for $N=200$. Long term evolution is stable, and the system has been succesfully evolved beyond $t=50,000 M$. 
$t=0.10$
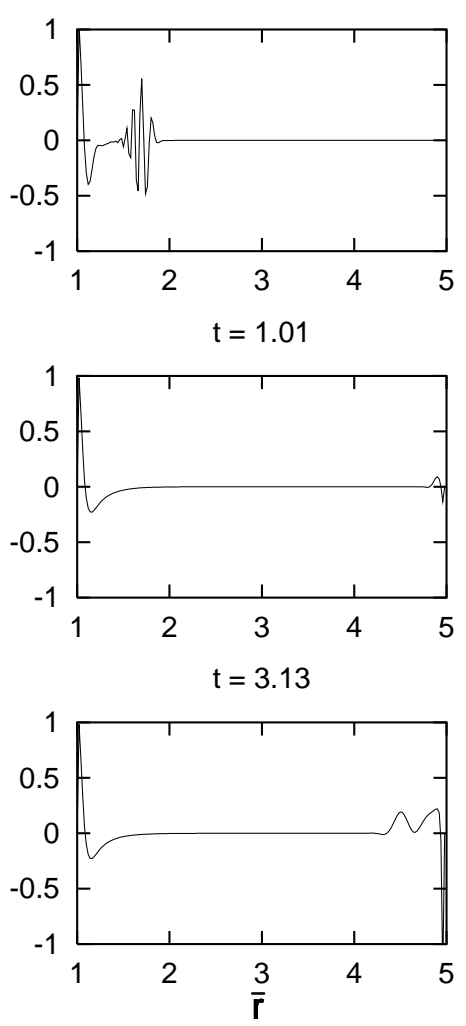

$t=0.30$
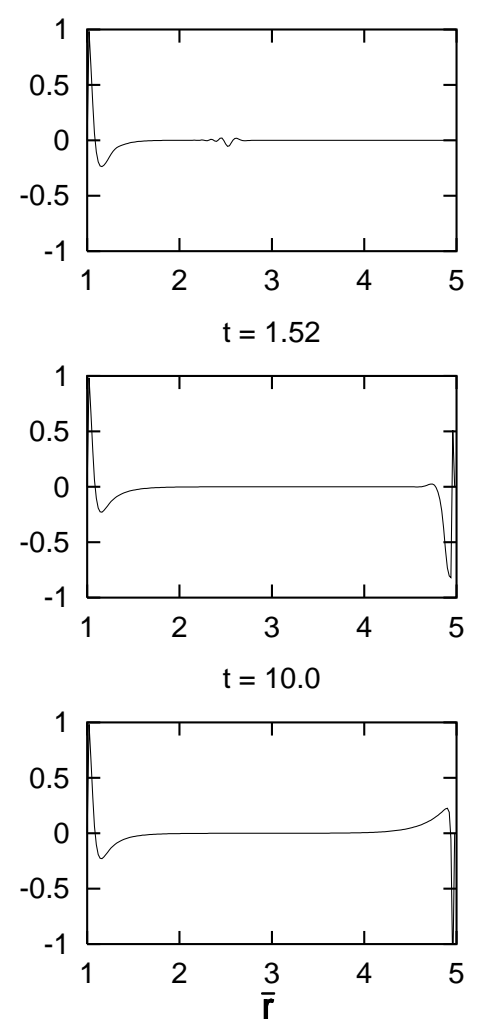

FIG. 9: Snapshots of the Hamiltonian constraint. For the same parameter choices as in Fig. 8 we display the Hamiltonian constraint during the early stages of evolution. A wave generated by truncation error at the inner boundary propogates outwards, is reflected by the outer boundary back into the domain, and then rapidly decays. No further evolution is seen in the Hamiltonian constraint after $t \approx 5$.

evolved stably using the naive Dirichlet boundary conditions and analytic lapse and shift conditions. Nevertheless, we have evolved such surfaces stably by incorporating (1) area locking shift conditions, (2) isometery conditions at the throat, and (3) an outgoing boundary condition based on the difference between the numeric and analytic solutions. These numerical results, and the corresponding stability analysis, are not within the scope of this paper and will be presented elsewhere. [1]

\section{FROM ONE BLACK HOLE TO TWO SPINNING BLACK HOLES}

In this paper we considered a static spacetime metric for the Schwarzschild black hole, with spacelike hypersurfaces of constant (not necessarily zero) value of the trace of the extrinsic curvature tensor. This slicing provides a natural generalization of the maximal slicing scheme currently in use in some numerical approaches to the binary black hole problem.

An essential feature of our $K$-constant metric is a spatially-varying radial shift vector, which allows the surfaces to avoid the singularity while evading the grid stretching problems often encountered with other metrics. Work is in progress to develop a geometric handle on our shift condition, akin to that of the "minimal distortion" shift often discussed. The inner and outer boundary conditions are also particularly convenient. In the inner regions, the "horizon hugging" feature of the $K$-slices, together with their regularity, may remove the need to excise the grid within the apparent horizon, thus providing a natural "boundary without a boundary" avoidance of the singularity. In addition, at the outer boundary the surfaces are asymptotically null, which may aid in gravity wave extraction.

In examining the characteristics of the $K$-slices we reviewed the three families of $K$-surfaces, including the horizonto-singularity $(H S)$ surfaces, as well as the more familiar horizon-to-horizon $(H H)$ and singularity-to-singularity $(S S)$ surfaces. All three families of surfaces either asymptote to the singularity, or to spatial infinity along a null surface. Thus the family of observers that make up such a foliation never "observe" the surfaces reach the singularity. This 
suggests a possible payoff in using the $H S$ surfaces in the evolution of black hole spacetimes, as there is no danger of the surface reaching the singularity. Just as there are natural boundary conditions which "freeze out" gravitational radiation as it progresses to null infinity, so too can we expect boundary conditions where the dynamic space freezes out as it approaches the singularity. Are there such natural boundary conditions at the future singularity? It would be worthwhile to investigate such asymptotically-null boundary conditions at the singularity of an $H S$ surface.

The future utility of numerical simulations of black hole spacetimes hinges in large part on a suitable choice of coordinates for the initial data, and on the particular evolution of these coordinates through the four lapse and shift conditions. These conditions are the only handles by which to manage the growth of the metric and curvature components during evolution. It is through judicious choices of lapse and shift that one is able to effectively enable singularity avoidance, and allow for efficient extraction of gravitational radiation. The simplistic example presented here may provide some guidance as to how to proceed in the more general case. $K$-surfaces in the generic two black hole problem can be expected to preserve the singularity-avoidance "horizon hugging" behavior, as well as remain asymptotically null at the boundaries. It remains to be seen what shift vectors are required to manage the growth of the intrinsic and extrinsic properties of the metric in the more general case, though the constant-crunch shift presented here is a natural starting point.

We are currently pursuing three avenues towards furthering and generalizing the work presented here. First, we are analyzing the stability of this coordinatization to small perturbations. Second, we are using the (1+1)-dimensional code discussed in the last section to examine a greater portion of the parameter space of initial data, so as to fully explore the numerical stability of the slicing. From our preliminary numerical investigations, we expect to be able to do a full spacetime "evolution" of Schwarzschild, and have it run stably and accurately for extended periods of time over a wide range of $H-K$ parameter space. Finally, we are analyzing the Oppenheimer-Snyder collapse in such $K$-slicings, following the lead of Eardley and Smarr, [8] as this will further test our slicing in a non static setting. [12]

\section{Acknowledgments}

We wish to acknowledge the Los Alamos National Laboratory LDRD/ER program for financial support. WAM wishes to thank the Institute for Theoretical Physics at UCSB for providing a stimulating working environment in which to complete part of this research. DH was supported in part by the National Science Foundation under Grant No. PHY9907949 to the ITP. PL was supported in part by NSF grants PHY9800973 and PHY9800970. We wish to thank Richard Matzner for advice on handling the coordinate ambiguity at the throat. We are especially grateful to John A. Wheeler for encouraging us to examine these slices in the context of the numerical treatment of black hole spacetimes.

[1] A.P. Gentle, D.E. Holz, P. Laguna, W. A. Miller, and D. Shoemaker, in preparation.

[2] M. Iriondo, E. Malec and N. Ó Murchadha, Phys. Rev. D54 (1996) 4792.

[3] J. Centrella and J. Wilson, Ap. J. Suppl. Series 54 (1984) 229.

[4] J. A. Wheeler, International J. Mod. Phys. A3 (1988) 2207-2247.

[5] F. J. Tipler and J. E. Marsden, Phys. Reports, 66 (1980) 109-139.

[6] F. J. Tipler and J. D. Barrow, Mon. Not. Roy. Astro. Soc., 216 (1985) 395-402.

[7] D. R. Brill, J. M. Cavallo and J. A. Isenberg, J. Math. Phys. 21 (1978) 2789.

[8] D. Eardley and L. Smarr, Phys. Rev. D19 (1978) 2239.

[9] A. Pervez, A. Qadir and A. Siddiqui, Phys. Rev. D51 (1995) 4598.

[10] M. Alcubierre, G. Allen, B. Bruegmann, T. Dramlitsch, J. A. Font, P. Papadopoulos, E. Seidel, N. Stergioulas, W. Suen, R. Takahashi, "Towards a Stable Numerical Evolution of Strongly Gravitating Systems in General Relativity: The Conformal Treatments," gr-qc/0003071.

[11] Richard Matzner, private communication, The University of Texas at Austin, March 20, 2000.

[12] Private communication at the 16th Pacific Coast Gravity Meeting, Caltech, March 24-25, 2000. 